\title{
Thermal diffusivity and conductivity of $\mathrm{Hg}_{1-x} \mathrm{Zn}_{x}$ Te solids and melts
}

\author{
Yi-Gao Sha, ${ }^{a)}$ Ching-Hua Su, K. Mazuruk, ${ }^{\text {a) }}$ and S. L. Lehoczky \\ Space Science Laboratory, NASA Marshall Space Flight Center, Huntsville, Alabama 35812
}

(Received 28 November 1995; accepted for publication 21 March 1996)

\begin{abstract}
The thermal diffusivity of pseudobinary $\mathrm{Hg}_{1-x} \mathrm{Zn}_{x} \mathrm{Te}$ solids and melts was measured by the laser flash method. The measured diffusivities for the solids of $0.10 \leqslant x \leqslant 0.30$ are about $60 \%$ of that of the HgTe solid. Those for the melts rise rapidly with temperature but less so with increasing $x$. For $x=0.30$, the diffusivity of the melt is about one third of that of the HgTe melt. Using the calculated heat capacity data from the associated solution model and measured density values, the thermal conductivity for the pseudobinary $\mathrm{Hg}_{1-x} \mathrm{Zn}_{x}$ Te solids of $0.10 \leqslant x \leqslant 0.30$ and for the melts of $x=0.10$, 0.16 , and 0.30 was determined. (C) 1996 American Institute of Physics. [S0021-8979(96)03413-5]
\end{abstract}

\section{INTRODUCTION}

Pseudobinary $\mathrm{Hg}_{1-x} \mathrm{Zn}_{x}$ Te solid solution is an important optoelectronic device material for infrared applications. The solid solution has a direct energy band gap which varies nearly linearly with composition $x$. In the growth of large $\mathrm{Hg}_{1-x} \mathrm{Zn}_{x} \mathrm{Te}$ crystals from the melt by the directional solidification method, it is very difficult to control and achieve the desirable growth interface shape due to the large interface segregation coefficient, the significant changes in the thermophysical properties upon the liquid-solid phase transformation, and the unavoidable radial temperature gradient near the interface. This radial temperature gradient is caused by the combination of a higher thermal conductivity in the melt than in the solid and the significant thermal conductivity of the ampoule compared to the sample resulted from the necessity to use a thick fused silica ampoule to contain the sample under extremely high mercury pressure at processing temperature. Thermal-fluid modelings of the growth system are indicative as how to achieve the desired interface shape but are not complete for lack of data on thermal conductivity of the sample as a function of temperature and composition.

Since pure fused silica has a thermal conductivity as high as that of a glass and the cross-section area of the ampoule wall is generally greater than that of the sample, the ampoule wall can constitute a large thermal sink in the sample-ampoule system. Therefore, the classic steady-state heat flow methods of thermal conductivity measurement are not practical. On the other hand, the similar property, thermal diffusivity, is not only an important thermophysical parameter in its own right, but it also offers a convenient, economical, and accurate method to derive the thermal conductivity.

A dynamic measurement system of the thermal diffusivity by the laser flash method ${ }^{1.2}$ has been employed previously to determine the thermal diffusivity of various composite materials. ${ }^{3}$ In this method the front surface of a small diskshaped sample is subjected to a very short burst of radiant energy from a laser pulse with a radiation time of $1 \mathrm{~ms}$ or less. The resulting temperature rise of the rear surface of the sample is measured and thermal diffusivity values calculated from the data of temperature rise versus time. Recently, ther-

\footnotetext{
${ }^{a)}$ Present address: Universities Space Research Association, Huntsville, AL.
}

mal diffusivities of $\mathrm{Hg}_{1-x} \mathrm{Cd}_{x} \mathrm{Te}$ solids and melts and those of pure $\mathrm{Te}$ and $\mathrm{Te}$-rich $\mathrm{HgCdTe}$ and $\mathrm{HgZnTe}$ melts were determined using the measurement system at Thermophysical Properties Research Laboratory (TPRL), West Lafayette, IN. ${ }^{4,5}$ This article describes similar measurements on $\mathrm{Hg}_{1-x} \mathrm{Zn}_{x}$ Te solids and melts.

\section{EXPERIMENT}

\section{A. Optical cell}

The optical cells were made of fused silica with two optical windows transparent to infrared wavelengths shorter than about $4 \mathrm{~mm}$. The cells were $16 \mathrm{~mm}$ outer diameter (OD) and $20 \mathrm{~mm}$ in overall thickness with a sidearm attached on the cylindrical side, which allowed the sample to be loaded and sealed. The disk-shaped cavity was $9 \mathrm{~mm}$ in diameter and 1-2 mm thick. The geometry of the cell can be found in Ref. 4.

Elemental $\mathrm{Hg}$ (7-9 grade), Zn (6-9 grade), and Te (6-9 grade, quadruple zone refined) from Johnson Matthey were loaded into the properly cleaned and outgassed optical cell and then sealed under vacuum of $10^{-5}$ Torr. The synthesis was carried out in a horizontal furnace with the sidearm placed horizontally to assure the contact of $\mathrm{Hg}$ with the other elemental pellets which were usually too large to slide into the cavity. The sample was heated to about $650^{\circ} \mathrm{C}$ over a period of several hours, held at this temperature for 3-5 days, and them cooled passively to room temperature. The cell was then placed with the sidearm vertical and the cavity downward in a vertical furnace. The sample was completely melted by raising the furnace temperature to about $900^{\circ} \mathrm{C}$ in $8 \mathrm{~h}$ and holding it for $16 \mathrm{~h}$. The cell cavity was usually filled by the melted sample with the help of shaking.

After cooling of the sample, the sidearm of the cell was then shortened and resealed and the cell reheated slowly to about $900^{\circ} \mathrm{C}$ to test its integrity. During this second heating procedure, several cells were observed to have developed or started to develop a crack near and parallel to the flat surface of the sample disk. It was later found that the crack formed during the heat up below and near the solidus temperature and it was concluded that the thermal expansion of the solid phase was the cause. To avoid the cell failure during the thermal diffusivity measurement, an alternative method of preparing the cells was adapted. The HgZnTe sample was 
TABLE $1 . H g_{1-x} \mathrm{Zn}_{x}$ Te optical cells for thermal diffusivity measurement. $L$ is the thickness of the sample disk.

\begin{tabular}{ccccc}
\hline \hline Cell name & $x$ & $L(\mathrm{~mm})$ & Total weight $(\mathrm{g})$ & Remark \\
\hline TZ10 & 0.10 & 1.558 & 1.1897 & Powder \\
TZ16 & 0.16 & 1.404 & 1.1716 & Powder \\
TZ20 & 0.20 & 1.577 & 1.2994 & Melted \\
TZ30 & 0.30 & 1.278 & 1.2098 & Powder \\
TZ40 & 0.40 & 1.772 & 1.3028 & Powder \\
\hline
\end{tabular}

first synthesized in another fused silica ampoule, collected afterwards by opening the ampoule, ground into powder, and reloaded and sealed into the optical cell. Enough material was prepared so that the optical cell cavity would be filled completely upon melting of the powder during the diffusivity measurements and all of the material in the synthesis ampoule was collected to assure the accuracy in sample composition. Table I lists optical cells prepared in this work for the thermal diffusivity measurement.

\section{B. Thermal diffusivity measurements}

During the measurements the optical cells were held vertically by two stainless-steel plates bolted tightly against the cell. Each plate, painted black to eliminate the scattering of the laser light, had a hole slightly smaller than the sample diameter and served as a beam stop assuring that no light beam bypasses the sample directly into the detector. The plates also prevented the cell from bursting in case of a cell failure. A split heater furnace with independent temperature control for the top and bottom elements was used to heat the samples. A ceramic tube was placed in the furnace coaxially with the sample and the detector to reduce the air convection caused by uneven heating. Such convection can cause fluctuation in the photopyrometer signal, making it difficult to determine the base-line temperature. The laser used was Korad K-2 pulsed neodymium glass laser with a wavelength of $1.06 \mu \mathrm{m}$. To limit the front face temperature rise, an attenuator consisting of a dilute aqueous solution of $\mathrm{CuSO}_{4}$ was sometimes applied. A mercury cadmium telluride (MCT) IR photopyrometer capable of detecting $0.05^{\circ} \mathrm{C}$ temperature change with a response time of milliseconds was employed to measure the temperature response of the rear face of the sample. The detector was coupled by an amplifier and an A/D converter to a computer-based data acquisition system which was crystal clock controlled. The computer collects the data, compares it with the corresponding theoretical curve, makes the corrections for heat loss by the ratio method of Clark and Taylor ${ }^{6}$ and by the thermal decay method of Cowan, ${ }^{7}$ and then plots the experimental and theoretical temperature rise curves. The thermal diffusivity can be calculated from the time needed for the rear face temperature rise to reach one half of its maximum value and the thickness of the sample. ${ }^{1,8}$

Because of the wide separation between the solidus and liquidus curves in the $\mathrm{HgZnTe}$ phase diagram, rapid heating was used when the sample temperature was between its solidus and liquidus and a mixing procedure was devised after the sample was completely melted. The cell containing the

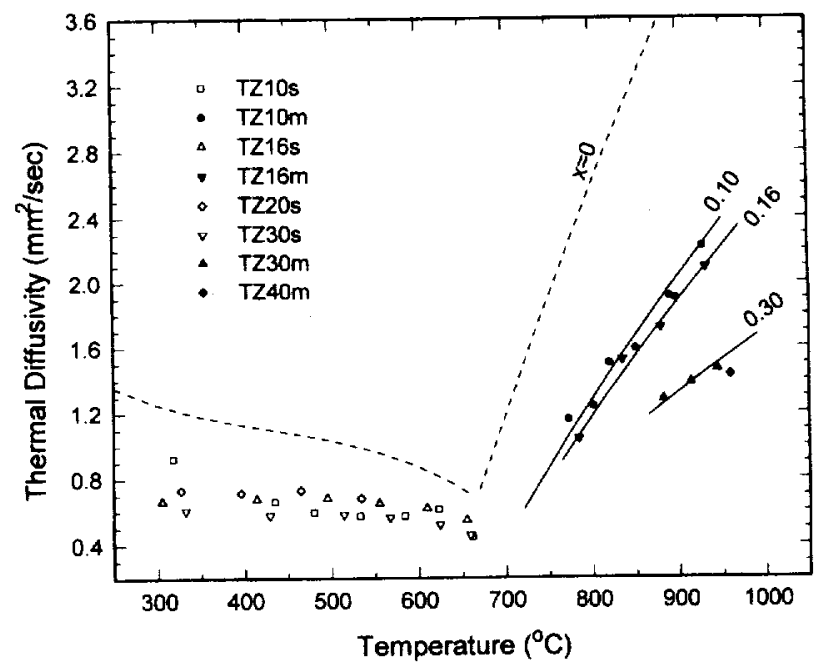

FIG. 1. Thermal diffusivity of pseudobinary $\mathrm{Hg}_{1-{ }_{x}} \mathrm{Zn}_{x} \mathrm{Te}$ solid solutions and melts. Symbols are measured data from this work. Solid lines are the best-fit curves for the diffusivity of melts for $x=0.10,0.16$, and 0.30 . The dashed lines are those of HgTe solid and melt, taken from Ref. 4.

melt was first brought to equilibrium in the furnace by holding the temperature for at least $20 \mathrm{~min}$ and then was pulsed with the laser at high power. The resulting $\mathrm{Hg}$ vapor pressure forces part of the cell content up into its stem which is directly above the cavity region. The cell was then observed to refill over the next few minutes. This procedure was repeated several times to ensure complete mixing of the sample. Inadvertent boiling was easily suppressed by applying a slight vertical temperature gradient. During cooling, a large vertical temperature gradient was applied to assure the sample was frozen from the bottom up. Rapid cooling was also essential to avoid the compositional segregation.

\section{RESULTS ON THERMAL DIFFUSIVITY}

Measurements were performed on five optical cells whose sample composition, thickness, and total weight are given in Table I. Cell TZ20 contained pre-melted sample and the measurements were carried out during heating. The cell ruptured after the measurement at $534{ }^{\circ} \mathrm{C}$ so that no data on melt were obtained. The other four cells contained powder samples and, therefore, were heated initially to the respective highest temperature of measurement to allow the samples to be completely melted and mixed. At these highest temperatures, the $\mathrm{Hg}$ partial pressure inside the cell was $70,75,80$, and $85 \mathrm{~atm}$ for cell TZ10, TZ16, TZ30, and TZ40, respectively. ${ }^{9}$ The measurements were made during the cooling for cells TZ10, TZ16, and TZ30. Cell TZ40 ruptured after the temperature had settled at $959^{\circ} \mathrm{C}$ for about $20 \mathrm{~min}$ yielding only one data point for the melt. The combined data are plotted in Fig. 1. Each data point is the average of 2-7 measurements made at that temperature with a standard deviation ranging from $5 \%$ to $15 \%$.

The measured thermal diffusivities for the pseudobinary $\mathrm{Hg}_{1-x} \mathrm{Zn}_{x}$ Te solids, $0.10 \leqslant x \leqslant 0.30$, are overlapping within the scatter of the data, especially near the solidus temperatures. Their temperature dependence is similar to those of the 
TABLE II. Parameters for the best fit to the thermal diffusivity of $\mathrm{Hg}_{1-\mathrm{x}} \mathrm{Zn}_{\mathrm{x}}$ Te melts. $D_{T}=A \ln T-B$, where $D_{T}$ is the thermal diffusivity in $\mathrm{mm}^{2} / \mathrm{s}$ and $T$ in ${ }^{\circ} \mathrm{C}$.

\begin{tabular}{cccc}
\hline \hline$x$ & $A\left(\mathrm{~mm}^{2} / \mathrm{s}\right)$ & $B\left(\mathrm{~mm}^{2} / \mathrm{s}\right)$ & $T_{\text {Liq }}\left({ }^{\circ} \mathrm{C}\right) \dagger$ \\
\hline 0 & $10.47^{\mathrm{a}}$ & $67.4^{\mathrm{b}}$ & 670 \\
0.10 & 6.43 & 41.7 & 721 \\
0.16 & 6.07 & 39.4 & 765 \\
0.30 & 3.62 & 23.3 & 865 \\
\hline
\end{tabular}

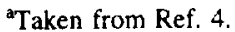

b Taken from Ref. 9.

$\mathrm{HgTe}$ and $\mathrm{HgCdTe}$ solids, ${ }^{4}$ showing a relatively strong decrease with increasing temperature below $400^{\circ} \mathrm{C}$ and above $600{ }^{\circ} \mathrm{C}$ and a relatively flat region in between. The range of values is also comparable to those of $\mathrm{HgCdTe}$ for the same $x$ values, being about $60 \%$ of those for the $\mathrm{HgTe}$ solid.

The data for the melt of $x=0.10$ are about the same magnitude as those of $\mathrm{Hg}_{0.9} \mathrm{Cd}_{0.1}$ Te melt but show a slightly weaker temperature dependence. For $x=0.30$, the measured thermal diffusivities are about one third of those of the $\mathrm{HgTe}$ melt and a smaller temperature dependence than those of the HdCdTe counterpart ${ }^{4}$ is observed here. The diffusivity data for the melt were fit by a logarithmic function for $x=0.10$, 0.16 , and 0.30 , respectively, and are shown by the solid curves in Fig. 1. Table II lists the best-fit parameters for these $x$ values.

\section{THERMAL CONDUCTIVITY}

The heat capacity for pseudobinary $\mathrm{Hg}_{1-x} \mathrm{Zn}_{x}$ Te melts have been calculated recently from an associated solution model by Su et al. ${ }^{10}$ For convenience, the original calculated values between the liquidus temperature and $1000^{\circ} \mathrm{C}$ for each $x$ value could be fit by a polynomial up to the fourth order. As has been described, the mass density of HgTe melt showed only a small temperature dependence. " A single average value of 8.038 was used here. The density of pseudobinary $\mathrm{Hg}_{1-x} \mathrm{Zn}_{x}$ Te melts was measured recently ${ }^{10}$ and can be approximated as 7.758 and 7.476 for $x=0.10$ and 0.16 , respectively. The three density data are linearly extrapolated to yield a value of 7.023 for $x=0.3$. Using the relation

$$
\sigma_{T}=\left(D_{T} C_{p} \rho\right) / M,
$$

where $\sigma_{T}$ is the thermal conductivity, $D_{T}$ the thermal diffusivity, $C_{p}$ the heat capacity per gram atom, $\rho$ the density and $M$ the gram-atomic weight, the thermal conductivity of the melts can be calculated, and the results are plotted in Fig. 2 . Because of the limited data measured for the thermal diffusi, ity and density, the thermal conductivity can only be obtained for three compositions and for a relatively small temperature range. Compared to the pseudobinary $\mathrm{Hg}_{1-x} \mathrm{Cd}_{x} \mathrm{Te}$ melt for $x=0.1$, the thermal conductivity obtained here for $\mathrm{Hg}_{0.9} \mathrm{Zn}_{0.1} \mathrm{Te}$ melt is about $25 \%$ lower at $800^{\circ} \mathrm{C}$ and has a noticeably weaker temperature dependence.

For the pseudobinary $\mathrm{Hg}_{1-x} \mathrm{Zn}_{x}$ Te solid solutions, a quasiregular solution model was validated by fitting comprehensive thermodynamic data, ${ }^{9}$ in which the excess free en-

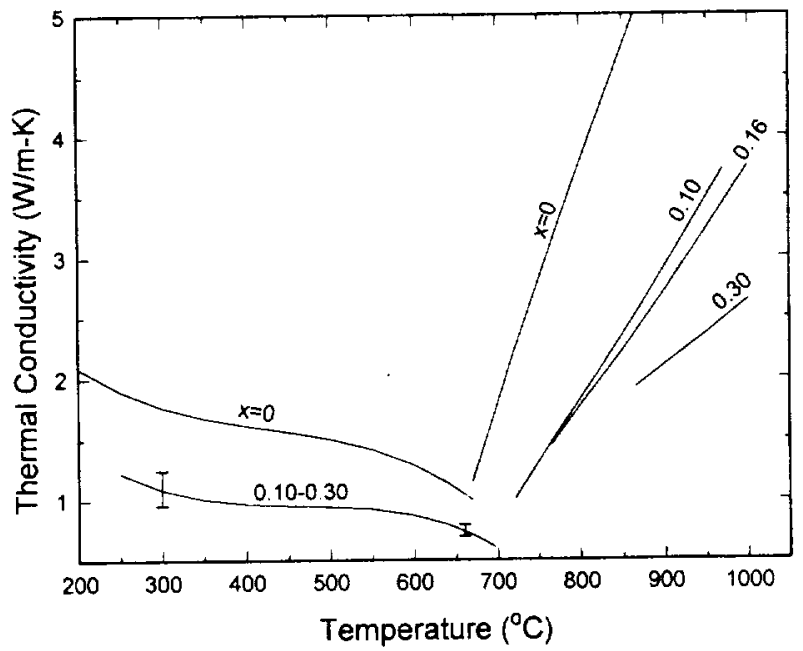

FIG. 2. Calculated thermal conductivity of pseudobinary $\mathrm{Hg}_{1}, \mathrm{Zn}_{x} \mathrm{Te}$ solids and melts.

ergy of formation, $\Delta G_{m}^{x s}$, depends only linearly on temperature. As a consequence, the heat capacity of the solid solution is given by

$$
C_{p}(x, T)=(1-x) C_{p}(\mathrm{HgTe}, T)+x C_{p}(\mathrm{ZnTe}, T),
$$

since any excess term is proportional to the second order derivative of $\Delta G_{m}^{x s}$ with respect to temperature. The heat capacities of $\mathrm{HgTe}$ and $\mathrm{ZnTe}$ can be expressed as the following: ${ }^{12,13}$

$$
\begin{aligned}
& C_{p}(\mathrm{HgTe}, T)=26.05+4.54 \times 10^{-3} \mathrm{~T}(\mathrm{~J} / \mathrm{g}-\mathrm{at} \cdot \mathrm{K}), \\
& C_{p}(\mathrm{ZnTe}, T)=23.22+5.44 \times 10^{-3} \mathrm{~T}(\mathrm{~J} / \mathrm{g}-\mathrm{at} \cdot \mathrm{K}),
\end{aligned}
$$

The density of the solid solution can be derived from the lattice parameter of the solid solution

$$
a_{0}=6.461-0.361 x(\AA)
$$

determined by Sher et al. ${ }^{14}$ and the molar weight of the solid solution. Although Eq. (5) is valid only at $298 \mathrm{~K}$, the error introduced by using it at $700^{\circ} \mathrm{C}$ is a few percent because the thermal expansion coefficient is in the order of $10^{-5}$.

The thermal diffusivity results obtained for the $\mathrm{Hg}_{1-x} \mathrm{Zn}_{x} \mathrm{Te}$ solid solutions of $x=0.10,0.16,0.20$, and 0.30 are scattering over each other and, for the calculation of thermal conductivity, a single curve was used to represent the combined data for $0.10 \leqslant x \leqslant 0.30$. Consequently, the calculated thermal conductivity becomes indistinguishable for such a composition range and one single curve is plotted in Fig. 2. The error bars near the end of the curve indicate the range corresponding to the upper and lower limit of the measured thermal diffusivity data. The results are comparable to those of the $\mathrm{Hg}_{1-x} \mathrm{Cd}_{x} \mathrm{Te}$ counterparts.

\section{DISCUSSION}

The thermal conductivity of the $\mathrm{Hg}_{1-x} \mathrm{Zn}_{x} \mathrm{Te}$ melts increases rapidly with the increasing temperatures. At $100^{\circ} \mathrm{C}$ above the liquidus temperature, $T_{\mathrm{liq}}$, it has values about $3-4$ times than those of the solids just below the solidus tempera- 
ture. As indicated by a theoretical calculation, ${ }^{15}$ a large radial temperature gradient can exist near the melt-solid interface in an insulating zone during the directional solidification growth if the melt and the solid thermal conductivities are significantly different and the sample container carries a significant amount of heat. This radial temperature gradient, combined with the significant changes in the thermophysical properties upon the liquid-solid phase transformation, causes the interface to bow towards the solid. The radial temperature gradient also causes gravity-driven convection in the melt which is often undesirable for the crystal growth.

The rapid increase in the thermal conductivity of the $\mathrm{Hg}_{1-x} \mathrm{Zn}_{x}$ Te melts results mainly from that of the thermal diffusivity (Fig. 1). In fact, the calculated heat capacity of the melts from the associated solution model decreases initially with increasing temperature ${ }^{10}$ and the measured density of the melts varies within $3 \%$ in the temperature range. ${ }^{10}$

\section{ACKNOWLEDGMENTS}

The authors wish to thank Raymond Taylor for providing the facility for the diffusivity measurement and Hans
Groot for technical assistant at TPRL. The work was supported by the Microgravity Science and Application Division of the National Aeronautics and Space Administration.

' W. J. Parker, R. J. Jenkins, C. P. Butler, and G. L. Abbott, J. Appl. Phys. 32, 1679 (1961).

${ }^{2}$ R. E. Taylor, High Temp.-High Pressures 11, 43 (1979).

${ }^{3}$ H. J. Lee and R. E. Taylor, J. Appl. Phys. 47, 148 (1976).

${ }^{4}$ L. R. Holland and R. E. Taylor, J. Vac. Sci. Technol. A 1, 1615 (1983).

${ }^{5}$ H. Maleki and L. R. Holland, J. Appl. Phys. 76, 4022 (1994).

${ }^{6}$ L. M. Clark and R. E. Taylor, J. Appl. Phys. 46, 714 (1975).

${ }^{7}$ R. D. Cowan, J. Appl. Phys. 34, 926 (1963).

${ }^{8}$ J. A. Cape and G. W. Lehman, J. Appl. Phys. 34, 1909 (1963).

${ }^{9}$ T.C. Yu and R. F. Brebrick, J. Phase Equilibria 13, 476 (1992).

${ }^{10}$ C.-H. Su, Y.-G. Sha, K. Mazuruk, and S. L. Lehoczky, J. Appl. Phys. (in press).

"D. Chandra and L. R. Holland, J. Vac. Sci. Technol. A 1, 1620 (1983).

${ }^{12}$ K. C. Mills, Thermodynamic Data for Inorganic Sulphides, Selenides, and Tellurides (Butterworths, London, 1974).

${ }^{13}$ I. Barin, O. Knacke, and O. Kubaschewski, Thermodynamical Properties of Inorganic Substances (Springer, Berlin, 1977).

${ }^{14}$ A. Sher, D. Eger, A. Zemel, H. Feldstein, and A. Raizman, J. Vac. Sci. Technol. A 4, 2024 (1986).

${ }^{15}$ R. J. Naumann and S. L. Lehoczky, J. Cryst. Growth 61, 707 (1983). 